\title{
Overdiagnosis and mistreatment of malaria among febrile patients at primary healthcare level in Afghanistan: observational study
}

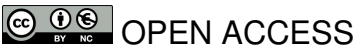

\begin{abstract}
Toby Leslie lecturer and project manager ${ }^{12}$, Amy Mikhail research fellow and project manager ${ }^{2}$, Ismail Mayan field research coordinator ${ }^{2}$, Mohammed Anwar field research coordinator ${ }^{3}$, Sayed Bakhtash field research coordinator ${ }^{4}$, Mohammed Nader technical coordinator ${ }^{3}$, Clare Chandler lecturer ${ }^{1}$, Christopher J M Whitty professor ${ }^{1}$, Mark Rowland reader and project leader ${ }^{1}$
\end{abstract}

${ }^{1}$ London School of Hygiene and Tropical Medicine, Keppel Street, London WC1E 7HT, UK; ${ }^{2}$ Health Protection and Research Organisation, Kabul, Afghanistan; ${ }^{3} \mathrm{HealthNet} \mathrm{TPO}$, Karte-Se, Kabul, Afghanistan; ${ }^{4}$ Medical Emergency Relief International, Kabul, Afghanistan

\begin{abstract}
Objective To assess the accuracy of malaria diagnosis and treatment at primary level clinics in Afghanistan.

Design Prospective observational study.

Setting 22 clinics in two Afghan provinces, one in the north (adjoining Tajikistan) and one in the east (adjoining Pakistan); areas with seasonal transmission of Plasmodium vivax and Plasmodium falciparum.

Participants 2357 patients of all ages enrolled if clinicians suspected malaria.

Interventions Established ( $>5$ years) microscopy (12 clinics in east Afghanistan), newly established microscopy (five clinics in north Afghanistan), and no laboratory (five clinics in north Afghanistan). All clinics used the national malaria treatment guidelines.
\end{abstract}

Main outcome measures Proportion of patients positive and negative for malaria who received a malaria drug; sensitivity and specificity of clinic based diagnosis; prescriber's response to the result of the clinic slide; and proportion of patients positive and negative for malaria who were prescribed antibiotics. Outcomes were measured against a double read reference blood slide.

Results In health centres using clinical diagnosis, although 413 of 414 patients were negative by the reference slide, 412 (99\%) received a malaria drug and $47(11 \%)$ received an antibiotic. In clinics using new microscopy, $37 \%$ (75/202) of patients who were negative by the reference slide received a malaria drug and $60 \%(103 / 202)$ received an antibiotic. In clinics using established microscopy, 50.8\% (645/1269) of patients who were negative by the reference slide received a malaria drug and $27.0 \%$ (342/1269) received an antibiotic. Among the patients who tested positive for malaria, 94\% (443/472) correctly received a malaria drug but only 1 of 6 cases of falciparum malaria was detected and appropriately treated. The specificity of established and new microscopy was $72.9 \%$ and $79.9 \%$, respectively. In response to negative clinic slide results, malaria drugs were prescribed to $270 / 905$ (28.8\%) and 32/154 (21\%) and antibiotics to $347 / 930$ (37.3\%) and 99/154 (64\%) patients in established and new microscopy arms, respectively. Nurses were less likely to misprescribe than doctors.

Conclusions Despite a much lower incidence of malaria in Afghanistan than in Africa, fever was substantially misdiagnosed as malaria in this south Asian setting. Inaccuracy was attributable to false positive laboratory diagnoses of malaria and the clinicians' disregard of negative slide results. Rare but potentially fatal cases of falciparum malaria were not detected, emphasising the potential role of rapid diagnostic tests. Microscopy increased the proportion of patients treated with antibiotics producing a trade-off between overtreatment with malaria drugs and probable overtreatment with antibiotics.

\section{Introduction}

Malaria remains one of the most important infectious diseases of poverty. Accurate diagnosis of the disease is essential for both targeting malaria drugs to those who need them and identifying those with alternative (often serious) non-malarial causes of infection. Consequently the use of malaria parasite based diagnostic tests using microscopy or rapid diagnostic tests lies at the heart of the World Health Organization's new global guidelines for the treatment of malaria. ${ }^{1}$ Clinical diagnosis of malaria, based on symptoms alone, is known to be inaccurate ${ }^{2-4}$ so diagnosis based on detection of parasites has clear advantages. In Africa, however, there is now clear evidence that overdiagnosis of malaria and misprescription of malaria treatment to patients who are negative for parasites is a widespread problem. As a consequence, serious non-malarial infections are missed, ${ }^{5-9}$ drugs are wasted, and the cost 
effectiveness of the diagnostic tests is reduced. ${ }^{10}{ }^{11}$ In south and central Asia, where programmes to expand access to malaria diagnosis are currently underway, this problem has been much less considered. This omission may be based on the assumption that no problem exists in south and central Asia, where the incidence of malaria is much lower and is responsible for a smaller proportion of febrile cases than in Africa. In south and central Asia, it has often been perceived that the greater risk is that cases of malaria are being missed rather than that malaria is being overdiagnosed and overtreated. Although the incidence of malaria in south and central Asia may be low compared with Africa, the population at risk is larger, ${ }^{12}{ }^{13}$ so the potential overall burden for public health if the misdiagnosis seen in Africa is also seen in south Asia is of considerable public health importance. The absence of data on overdiagnosis of malaria in this part of Asia is therefore an important gap, and any assumption that problems found in Africa are not relevant to Asia is probably fallacious and certainly needs substantiating. In most of south and central Asia malaria is predominantly caused by Plasmodium vivax, but Plasmodium falciparum is also seen. ${ }^{13}$ This presents distinct operational challenges, different from Africa and South East Asia where most or almost all malaria is caused by $P$ falciparum and transmission is generally more intense: firstly, among the causes of fever, malaria is in the minority so needs to be differentiated from non-malarial fevers, and, secondly, the rarer and more pathogenic $P$ falciparum needs to be differentiated from $P$ vivax to allow proper targeting of artemisinin combination therapy. Overdiagnosis of malaria results in other potentially serious infections being overlooked, such as pneumonia and invasive bacterial disease. ${ }^{14-16}$ Although mortality from vivax malaria in South East Asia is more common than previously thought, ${ }^{17}$ it is low compared with falciparum malaria or other bacterial causes of febrile illness, so the risk to the patient if malaria is missed is lower than in areas dominated by falciparum malaria. Missing a serious non-malarial infection because of overtreatment of malaria is therefore a greater threat to people and likely to be more wasteful of resources in this setting than in Africa. Microscopy is the standard method of parasite based malaria diagnosis. ${ }^{19}$ At the periphery of stable countries and in crisis affected areas, however, most people do not have access to accurate parasitological diagnosis and treatment is largely based on clinical signs and symptoms.

Since so few data are available from south or central Asia we undertook an observational study to determine the accuracy of diagnosis and targeting of treatment in 22 clinics at primary healthcare level. The clinics were in malaria endemic areas of Afghanistan where either clinical or microscopic diagnosis of malaria was routinely applied and where falciparum and vivax malaria coexist.

Afghanistan is an important setting to deal with the problem of overdiagnosis and mistreatment of malaria. It is situated in the WHO Eastern Mediterranean Region, which has designs on the elimination of malaria as a medium to long term goal; it borders the WHO-European Region area, which aims to be malaria free by 2015 ; acute febrile illnesses are a major cause of morbidity and mortality; case management is a major public health problem; and resources are scarce. The health services of the country are being rebuilt and coverage of services expanded, so evidence that improves policy and practice is important at this stage of Afghanistan's development to prevent entrenchment of poor practice.

\section{Methods \\ Study area, health system, and sites}

The study was carried out in two Afghan provinces, one in the north (adjoining Tajikistan) and one in the east (adjoining Pakistan). In both provinces malaria is a minor cause of febrile illness. In the north, low transmission results in a slide positivity rate of less than $1 \%$, whereas in the east transmission is more intense and the slide positivity rate reaches $10-30 \%$. $P$ vivax is the predominant species, accounting for $80-90 \%$ of malaria cases annually; the remainder being caused by $P$ falciparum. Transmission is seasonal, limited by altitude and temperature, with falciparum malaria occurring in late summer and autumn and vivax malaria in spring and summer. ${ }^{19-21}$ Malaria control, focused on the distribution of insecticide treated nets amid improving post-conflict healthcare coverage, has succeeded in reducing transmission in Afghanistan so malaria is now less common than it was 5-10 years before this study took place.

The health system in which the study took place has a well defined hierarchical structure (the Basic Package of Health Services) and has been a priority of post-conflict development, implemented by non-governmental organisations. ${ }^{22}$ The Basic Health Centres and Comprehensive Health Centres in the study are the primary points of access for free outpatient services.

The health system structure is comparable in each province but differs in the availability of malaria diagnosis. In the eastern region most health facilities have microscopy, which was established 10-20 years ago. In the northern region microscopy had not been established before 2009, but during the study five laboratories were established by the national programme and equipped with microscopy. This allowed the comparison of targeting diagnostic accuracy and treatment in three contrasting operational settings. In the north (low transmission of malaria), five clinics used clinical diagnosis (with no laboratory support) and five had newly established microscopy (August 2009), whereas in the east (high transmission of malaria) all 12 clinics had been using microscopy for at least five years (table $1 \Downarrow$ ).

In routine care the treatment of malaria is based on the national malaria treatment guidelines. Under the guidelines a combination of chloroquine and sulfadoxine-pyrimethamine is used to treat clinically diagnosed malaria (termed suspected malaria in the guidelines), chloroquine is used to treat laboratory confirmed vivax malaria (termed confirmed vivax), and artemisinin combination therapy (sulfadoxine-pyrimethamine with artesunate) is used to treat laboratory confirmed uncomplicated falciparum malaria (termed confirmed falciparum). The guidelines do not provide instruction on treatment or further examination of patients with a negative diagnosis.

Consultations are carried out by trained doctors or, in their absence, by nurses or midwives. In this study we describe any health workers who carried out consultations with and prescribed medicine to patients as clinicians.

The selection of health centre was based on location; we considered basic health centre and community health centre clinics eligible if they were located in secure areas, accessible to study staff, and did not routinely refer patients to an external laboratory for diagnosis. Based on these criteria, we excluded 36 clinics in the study provinces. Overall, 10 clinics were selected in the north and 12 in the east (table 1).

\section{Patient enrolment}

Trained study staff (registrars) screened patients who presented at the clinics with non-specific self reported fever. These patients were then evaluated by the clinician against the inclusion and 
exclusion criteria. Inclusion criteria were any patient where the clinician considered malaria in the diagnosis (either prescribing a malaria drug or requesting a diagnosis, or would request a diagnosis if it were available) and the patient (or parent or guardian) gave informed consent. Exclusion criteria were patients with a diagnostic result from another clinic, those who the clinician referred to another facility for diagnosis, and those in a critical condition or requiring urgent treatment. Eligible patients were invited to give written informed consent and enrolled.

\section{Data collection}

Clinic data were recorded on proforma patient tracking forms. After enrolment the study registrar noted the patient's personal details and the clinician recorded the patient's signs and symptoms, the final diagnosis (based on clinical signs or the results of the slide), and the treatment prescribed.

In clinics where microscopic diagnosis was available, the clinician was given the result of the clinic slide (read by the clinic microscopist) in 30-60 minutes and the result was noted on the form. The information on the form included the species, parasite stages, and, if carried out, parasite counts. This process matched (and was part of) the normal operations in the clinics. Data were double entered using Microsoft Access 2007 (Microsoft, Seattle, WA).

\section{Study outcomes}

We compared the operational effectiveness of the three diagnostic interventions in providing accurate diagnosis and treatment of malaria. The primary outcome was the proportion of patients with suspected malaria who were accurately treated for malaria. This proportion was defined as patients who were positive for malaria parasites by reference slide and received an appropriate malaria drug and patients who were negative by reference slide and did not receive a malaria drug and were therefore independent of the slide positivity rate.

Secondary outcomes were the accuracy of clinic based microscopy compared with the reference slides, the proportion of patients with positive and negative results for malaria by clinic slide who were prescribed a malaria drug, the proportion of patients with negative results by clinic slide who received an antibiotic, and the proportion of patients positive for $P$ falciparum who received artesunate combination therapy.

\section{Statistical analysis}

Enrolment in 2009 covered the period from June to September, when the transmission seasons for $P$ vivax and $P$ falciparum concur. The sample size calculation was based on a slide positivity rate of $20 \%$ (not met owing to the decreasing prevalence of malaria in febrile patients) and aimed for a target of 1980 patients per arm. We used logistic regression analysis to identify associations between preselected explanatory variables: diagnostic type, positive or negative reference slide result for malaria, sex, age group, status of clinician (doctor $v$ nurse or midwife), clinic type (Comprehensive Health Centre or Basic Health Centre), and the primary outcome of accurate treatment. We made no additional correction for clustering. Diagnostic accuracy was measured using the standard measures of sensitivity and specificity. Data were analysed using Stata v11.

\section{Sample collection and laboratory methods}

Blood slides were collected at all sites. In health centres with no microscopy, trained study registrars prepared slides onsite for reading at the reference laboratory. In health centres with microscopy, the microscopist prepared routine slides for the clinic diagnosis and collected a second slide for storage as a reference slide.

Both thick and thin smears were prepared. These were air dried, fixed with methanol, and stained with $10 \%$ Giemsa solution for 30 minutes. The clinic microscopist read the clinic slides and fed back results to the clinician. The clinic microscopists received no additional training before the study.

The clinic microscopist (study registrar if in a clinic using clinical diagnosis) prepared and fixed the reference slide before storage. The expert microscopist in either the north or east stained these slides for a first read within three days of being fixed. The slides were then transported to Kabul for a second (and third) reading. The expert microscopists double read the reference slides at $150 \times$ magnification, blinded to each other's results and to that of the clinic slide (if taken). A slide was declared negative if no parasites were seen after examination of 100 fields. If a slide was positive, a parasite count was carried out and quantified against 200 white blood cells. If the diagnosis of the two microscopists differed, a third microscopist examined the slide and a best of three rule was applied.

\section{Results}

A total of 2381 patients were enrolled in the study from 1 July to 19 September 2009 (figure $\Downarrow$ ). Table $2 \Downarrow$ shows the characteristics of the sample. Data from 24 (1.0\%) patients were not evaluable because of missing reference slides or failure to record the final diagnosis or treatment.

Few patients in the north had malaria according to the reference slide result: only one of $415(0.2 \%)$ in the clinical diagnosis arm and none of 202 in the new microscopy arm (table 3, $\Downarrow$ figure). More patients had malaria in the east: 471 of 1740 (27.1\%) were positive for malaria-six $(1 \%)$ of the 471 positive cases had falciparum infection and 465 (99\%) had vivax infection. One of these cases was coinfected with falciparum and vivax malaria.

\section{Primary outcome analysis}

In health centres in the north that used clinical diagnosis, only $1 \%(3 / 415)$ of patients were accurately treated for fever (table $3)$. In health centres in the north that used new microscopy, treatment accuracy was 63\% (127/202) and in health centres in the east where microscopy was established, treatment accuracy was $60.7 \%\left(1056 / 1740, \chi^{2}=0.4, \mathrm{P}=0.5\right)$.

Most patients who were classified as inaccurately treated $(97 \%)$ had been prescribed a malaria drug but had a negative reference slide result. Among the 414 who were clinically diagnosed, 412 (99\%) had a negative reference slide result but were treated with a malaria drug (table 3, figure). In the new microscopy setting, this proportion was $37 \%(75 / 202)$ compared with $50.8 \%$ $(645 / 1269)$ in the established microscopy setting $\left(\chi^{2}=51.5\right.$, $\mathrm{P}<0.001)$.

The logistic regression analysis showed that diagnosis by microscopy was the main factor associated with accurate treatment of patients suspected of having malaria (table $4 \Downarrow$ ). Other factors associated with accurate treatment were being treated by a midwife or nurse rather than a doctor and being treated in a community health centre rather than a basic health centre. Children aged 6-10 years were less likely to be accurately 
treated than other age groups (adjusted odds ratio 0.6, 95\% confidence interval 0.4 to $0.8 ; \mathrm{P}<0.001$ ). Those treated in the centres with new microscopy were more likely to be accurately treated than those in the centres with established microscopy $(2.2,1.5$ to $3.2 ; \mathrm{P}<0.001)$.

\section{Accuracy of clinic microscopy}

Both clinic based microscopy settings had a low specificity. In the clinics in the north with new microscopy, there were 39/193 (20\%) false positive results, giving a specificity of $79.8 \%$ (table $5 \Downarrow)$. In the clinics in the east with established microscopy, there were $329 / 1212(27.1 \%)$ false positive results $\left(\chi^{2}=2.8, \mathrm{P}=0.1\right)$. In the higher endemic eastern area, where more malaria cases were recorded, there were 47/451 (10\%) false negative results, giving a sensitivity of $89.6 \%$ and a specificity of $72.9 \%$. Clinical diagnosis in the low transmission setting had a specificity of $0.2 \%$, with $412 / 413(99 \%)$ false positive results.

\section{Clinicians' response to diagnosis}

Among the health centres with microscopy, a blood slide was produced in the clinic for most patients $(95.6 \%)$. In the new microscopy arm, malaria treatment was given to $37 / 39$ (95\%) patients with a positive clinic slide result compared with $732 / 733$ (99.9\%) in the established microscopy arm (Fisher's exact $\mathrm{P}=0.007)$. Overall, 21\% (32/154) of patients with a negative clinic slide result were treated with a malaria drug in the new microscopy arm compared with 270/905 (28.8\%) in the established microscopy arm $\left(\chi^{2}=5.3, \mathrm{P}=0.02\right)$.

Doctors in the established microscopy arm were more likely to prescribe a malaria drug in the presence of a negative clinic slide result than were nurses or midwives $(254 / 795,31.9 \% \mathrm{v}$ 16/140, 11\%; Fisher's exact test: $\mathrm{P}<0.001$ ) (table $6 \Downarrow$ ), although the type of patient (age, sex) did not differ. This trend was less evident in the new microscopy arm (25/109 23\% v 7/45 16\%; Fisher's exact test: $\mathrm{P}=0.3$ ) (table 6 ). For patients with a positive clinic slide result, nurses and midwives prescribed malaria drugs with near $100 \%$ accuracy.

\section{Prescription of artemisinin combination therapy for falciparum malaria}

Reference slides were positive for $P$ falciparum for six patients, giving a slide positivity rate in the east of $0.3 \%$ (6/1740). Only one slide was correctly identified by the clinic microscopist as containing $P$ falciparum and the patient was treated with artemisinin combination therapy. Of the four undiagnosed cases of falciparum monoinfection, two were diagnosed as negative and two were diagnosed as containing vivax by clinic microscopy. The two patients who were wrongly diagnosed as having vivax malaria were given chloroquine, one of those declared negative was given sulfadoxine-pyrimethamine with chloroquine (the recommended treatment for suspected malaria) and the other received no malaria drug.

Clinic microscopists identified 14 cases of falciparum malaria. Compared with the reference slide, nine of these were negative, four had $P$ vivax, and one had $P$ falciparum. Of these 14 patients, three were treated with chloroquine and six with sulfadoxine-pyrimethamine, one was indeterminate, and four were prescribed doses of artemisinin combination therapy (sulfadoxine-pyrimethamine with artesunate). One dose of artemisinin combination therapy was correctly given to the patient with accurately detected falciparum malaria and to three patients with clinic slides that were false positive for falciparum malaria (two were negative and one was positive for vivax malaria by the reference slide).

\section{Prescription of antibiotics}

Antibiotics were prescribed to $20.8 \%$ (362/1740) of patients with suspected malaria in the clinics with established microscopy and $60 \%(103 / 202)$ in the clinics with new microscopy $\left(\chi^{2}=90.6\right.$, $\mathrm{P}<0.001)$. The percentage of patients receiving antibiotics was lower $(11 \%, 47 / 414)$ in the clinical diagnosis arm compared with established microscopy arm $\left(\chi^{2}=19.4, \mathrm{P}<0.001\right)$.

In response to negative clinic slide results clinicians prescribed antibiotics to $64 \%(99 / 154)$ of patients in the new microscopy arm and 37.3\% (347/930) in the established microscopy arm $\left(\chi^{2}=302 ; \mathrm{P}<0.001\right)$. By comparison, 270/905 (28.8\%) of patients with a negative clinic slide result received a malaria drug in the established microscopy arm $\left(\chi^{2}=11.5 \mathrm{P}=0.001\right)$. Antibiotics were less frequently prescribed to patients with positive clinic slide results than negative in both microscopy arms but there were more prescriptions for cotreatment with antibiotics and malaria drugs in the new microscopy arm than in the established microscopy arm $(8 \%(3 / 29) v 1.9 \%(14 / 733)$, respectively, $\left.\chi^{2}=5.7 ; \mathrm{P}=0.02\right)$.

Of 512 prescriptions of antibiotics, the most frequently prescribed were amoxicillin $(181,20.5 \%)$, penicillin $(127,24 \%)$, cotrimoxazole $(105,20.5 \%)$, chloramphenicol $(30,6 \%)$, doxycycline $(13,2.5 \%)$, and ciprofloxacin $(5,1 \%)$. Tonsillitis followed by urinary tract infections were the most common alternative diagnoses given by the clinicians for patients with a negative test result.

\section{Variation between clinics}

The accuracy of treatment varied significantly between microscopy clinics. In the new microscopy arm, the accuracy of treatment ranged from $7 \%$ to $86.2 \%$. In the established microscopy arm, the accuracy of treatment in basic health centres ranged from $37 \%$ to $68 \%$ and in community health centres from $51.3 \%$ to $91.5 \%$. Among the clinics with clinical diagnosis, accuracy was uniformly poor $(<1 \%)$.

\section{Discussion}

Our study shows that in this south Asian setting of low incidence of malaria, almost all of which is the relatively less serious vivax form, malaria is substantially overdiagnosed as a cause of infection and results in large numbers of patients with other causes of acute febrile illness being mistreated. The few cases of falciparum malaria seen in this epidemiological setting were mostly not detected and consequently were not treated with artemisinin combination therapy. Because malaria is much less common and predominantly due to Plasmodium vivax, overtreatment of malaria presents a worse risk-benefit outcome to patients than it would in Africa, or some areas of South East Asia where a higher proportion of febrile illness is due to malaria and is mostly caused by the potentially fatal Plasmodium falciparum.

\section{Diagnosis and drug targeting}

Compared with clinical diagnosis, microscopy improves the targeting of malaria drugs, but only by half, and it increases the prescription of antibiotics. Problems with both the quality of microscopy and the prescribers' response to the diagnostic result, equally contribute to a cumulative $40-50 \%$ loss of accuracy in treatment for malaria. Microscopy in both epidemiological settings produced a high proportion of false positive diagnoses and, as in Africa ${ }^{83-25}$ and a single centre study from India, ${ }^{16}$ clinicians often disregarded negative diagnostic results and provided malaria treatment. Comparison between new and 
established microscopy suggests that the problem occurs in both entrenched and new microscopy services in the primary health system. Since the population at risk of malaria in the south and central regions of Asia is larger than that in Africa, ${ }^{12}{ }^{13}$ and acute infection remains a major part of avoidable mortality and morbidity, the public health implications are important.

Although most patients with malaria received a malaria drug, targeting of artemisinin combination therapy to those with confirmed falciparum malaria was poor. This was chiefly because of inaccurate microscopy resulting in undetected infections and false positive results. This shows the challenge of accurately identifying rare cases of falciparum malaria in a transmission area where vivax malaria is predominant. Even when clinicians were given a clinic slide result (rightly or wrongly) mentioned as containing falciparum, less than $30 \%$ of the affected patients were treated with artemisinin combination therapy, suggesting that targeting would be poor even if microscopy was accurate.

Antibiotic prescription was more common where microscopy rather than clinical diagnosis was used and was more common among patients with a negative clinic slide result than positive. Even so, patients with a negative clinic slide result were almost as likely to be treated with a malaria drug as with an antibiotic and, overall, patients with a negative reference slide result were nearly twice as likely to receive a malaria drug than an antibiotic. In low transmission areas where clinical diagnosis was used, the chances of malaria were less than $1 \%$, but prescription of antibiotics was infrequent and almost all patients were treated with malaria drugs. Although data on the causes of non-malarial fever in Afghanistan is scant, ${ }^{26}$ most cases may be viral and self limiting (although bacterial causes cannot be ruled out ${ }^{27}$ ), might respond to antibiotics, and are undoubtedly being missed. In Tanzania and Kenya, for example, around $10 \%$ of children admitted to a hospital had pathogenic bacteria detected, most being non-typhi salmonella and pneumococcus ${ }^{14}{ }^{15}$ in patients who were both positive and negative for malaria, but in the same sites under $2 \%$ of outpatients who were negative for malaria and almost no outpatients who were positive for malaria had bacteraemia. ${ }^{28-30}$ Patterns of bacterial sepsis are different between Africa and Asia (for example, typhoid is significantly more common in south Asia) so it is not possible to say whether the increase in antibiotic prescription seen here represents over-prescription or better targeting of antibiotics. The choice of antibiotics by clinicians would, however, not be ideal for covering Gram negative sepsis. Antibiotics are over prescribed in most healthcare settings, and from a public health perspective the improved treatment of malaria may be offset by the negative effects of overtreatment with antibiotics. This will be resolved by studies with reliable bacteriology (not currently available in this low resource setting).

\section{Improved targeting}

The question of how to treat patients when test results are negative is a common dilemma for clinicians in malaria endemic countries. ${ }^{5}$ In studies in east and west Africa, ${ }^{7831} 32$ the decision to treat patients who have negative test results with malaria drugs is borne out of several factors: an inflated perception of malaria risk compared with the real level of transmission, the lack of alternative treatments for febrile illness, and low confidence in negative slide results. ${ }^{31}{ }^{32}$ Guidelines themselves contribute to the problem by adopting a precautionary principle or appearing ambiguous in their definition of "confirmed" and "suspected" or "clinical" malaria. ${ }^{31-33}$ Adherence to negative diagnostic test results differs significantly between doctors and nursing staff even though both groups of practitioners use the same guidelines. This leads to highly variable quality of service from clinic to clinic, reinforcing the role of quality assurance, unambiguous guidelines, and standardised clinical training.

Improving and sustaining coverage of quality microscopy services is challenging in under-resourced health systems. ${ }^{34}$ In areas of low transmission, detection of the relatively rare cases of malaria among febrile patients is crucial for sustaining control and progression to elimination because systematic overdiagnosis exaggerates the real burden of malaria. Rapid diagnostic tests for malaria are now being widely deployed and if their accuracy and quality can be maintained under routine conditions they may improve diagnostic accuracy at the clinic level. ${ }^{35} 25$ However, if the deployment is not accompanied by changes in treatment practice by clinicians, then rapid diagnostic tests will not reach their full potential. Evaluation of the impact of such tests on differential diagnosis and treatment of vivax and falciparum malaria from non-malarial fevers in south and central Asia should be a priority, and improving the standards of existing microscopy requires more investment.

\section{Limitations and applicability to other areas}

This study has the limitations of any observational study. There is potential for a Hawthorne effect-where clinicians' behaviour may be altered by observation. If applicable here, this effect would probably result in an improved performance and an underestimate of the scale of the problem. Although new microscopy was introduced into the northern region of Afghanistan by the national malaria control programme (and not the investigators) during the study, it was a non-random introduction and in view of this and due to other differences between the microscopy settings (for example, intensity of transmission) we have been cautious in the interpretation of differences between new and established microscopy.

Our findings are likely to be applicable to other areas of Afghanistan. As the study area and health system were not unlike much of malaria endemic Afghanistan, we consider it likely to be representative of practice in the country as a whole. Despite Afghanistan's well documented poverty and chronic conflict, healthcare reconstruction has received impressive investment and political support and is run by contracted non-governmental organisations on a performance basis. ${ }^{36} \mathrm{In}$ most of south and central Asia, by comparison, public health systems are often equally or more impoverished but have not received the same levels of investment as their Afghan counterparts so, contrary to many people's assumptions, this part of Afghanistan may have better health services than other areas of south and central Asia that serve impoverished communities (services for urban higher socioeconomic groups are often better and provided privately, but this group is less likely to get malaria). Overdiagnosis was also common in the Indian study from a relatively well resourced (single) health centre. ${ }^{16}$ Until widespread misdiagnosis and treatment has been corroborated in other countries it is impossible to be sure that it is representative of south and central Asia in general, but our view is that it is safer to assume that it is and to take robust steps to identify and tackle what is likely to be a major public health challenge.

\section{Conclusion}

Despite the low incidence of vivax malaria in Afghanistan, there is consistent, substantial overdiagnosis of non-malarial fevers as malaria, falciparum malaria is poorly targeted with artemisinin combination therapy, and severe bacterial illnesses are probably being overlooked. When parasite based diagnosis 
is used, a reduction in overtreatment with malaria drugs is achieved but this gain may come at the cost of over-prescription of antibiotics. Since acute infections are such a large part of the burden of avoidable mortality and morbidity and the population of affected areas so large, this is a major public health problem. Improvements in the quality and coverage of diagnosis, evidence based guidelines for the diagnosis of other causes of febrile illness, and provision of alternative evidence based treatments for patients with negative test results for malaria are required. However, efforts to improve diagnostic coverage and accuracy will be undermined without concurrent interventions to change understanding, behaviour, and practice among clinicians.

Contributors: TL, AM, MR, CC, and CJMW designed the study. IM, MA, $\mathrm{SB}$, and NM assisted in design, trained the field workers, managed the study in the field, and contributed to data analysis and interpretation. $\mathrm{TL}$ analysed the data, wrote the initial draft, and is the guarantor of the study. All authors contributed to the final manuscript and approved the submitted version. All authors had full access to all of the data and take responsibility for the integrity of the data and the accuracy of the data analysis.

Funding: The study was funded by a grant from the Bill and Melinda Gates Foundation as part of the ACT Consortium (www.actconsortium. org) and sponsored by the London School of Hygiene and Tropical Medicine. Neither the funder nor sponsor had any role in the design or conduct of the study, analysis or interpretation of the data, or in the decision to publish.

Competing interests: All authors have completed the ICMJE uniform disclosure form at www.icmje.org/coi_disclosure.pdf (available on request from the corresponding author) and declare: no support from any organisation for the submitted work; no financial relationships with any organisations that might have an interest in the submitted work in the previous three years; and no other relationships or activities that could appear to have influenced the submitted work.

Ethical approval: The study protocol was approved by the Institutional Review Board of the Ministry of Public Health, Islamic Republic of Afghanistan (No 112453) and by the ethics review board of the London School of Hygiene and Tropical Medicine (No 5386).

Data sharing: No additional data available at this time but queries regarding sharing of data, protocols, etc should be addressed to the corresponding author (toby.leslie@lshtm.ac.uk).

1 WHO. Guidelines for the treatment of malaria. 2nd ed, 2010. 2012. www.who.int/malaria/ publications/atoz/9789241547925/en/index.html.

2 Chandramohan D, Jaffar S, Greenwood B. Use of clinical algorithms for diagnosing malaria. Trop Med Int Health 2002:7:45-52.

3 Luxemburger C, Nosten F, Kyle DE, Kiricharoen L, Chongsuphajaisiddhi T, White NJ. Clinical features cannot predict a diagnosis of malaria or differentiate the infecting species in children living in an area of low transmission. Trans $R$ Soc Trop Med Hyg 1998;92:45-9.

4 Weber MW, Mulholland EK, Jaffar S, Troedsson H, Gove S, Greenwood BM. Evaluation of an algorithm for the integrated management of childhood illness in an area with seasonal malaria in the Gambia. Bull World Health Organ 1997;75(Suppl 1):25-32

5 Whitty CJ, Chandler C, Ansah E, Leslie T, Staedke SG. Deployment of ACT antimalarials for treatment of malaria: challenges and opportunities. Malar J 2008;7(Suppl 1):S7.

6 Bisoffi Z, Gobbi F, Angheben A, Van den Ende J. The role of rapid diagnostic tests in managing malaria. PLoS Med 2009;6:e1000063.

7 Nankabirwa J, Zurovac D, Njogu JN, Rwakimari JB, Counihan H, Snow RW, et al. Malaria misdiagnosis in Uganda-implications for policy change. Malar $J 2009 \cdot 8 \cdot 66$

8 Hamer DH, Ndhlovu M, Zurovac D, Fox M, Yeboah-Antwi K, Chanda P, et al. Improved diagnostic testing and malaria treatment practices in Zambia. JAMA 2007;297:2227-31.

9 A-Elgayuom SM, El-Feki Ael K, Mahgoub BA, El-Rayah el A, Giha HA. Malaria overdiagnosis and burden of malaria misdiagnosis in the suburbs of central Sudan: special emphasis on artemisinin-based combination therapy era. Diagn Microbiol Infect Dis 2009;64:20-6.
10 Lubell Y, Reyburn H, Mbakilwa H, Mwangi R, Chonya S, Whitty CJ, et al. The impact of response to the results of diagnostic tests for malaria: cost-benefit analysis. $B M J$ 2008:336:202-5.

11 Zurovac D, Larson BA, Skarbinski J, Slutsker L, Snow RW, Hamel MJ. Modeling the financial and clinical implications of malaria rapid diagnostic tests in the case-management of older children and adults in Kenya. Am J Trop Med Hyg 2008;78:884-91.

12 Hay SI, Okiro EA, Gething PW, Patil AP, Tatem AJ, Guerra CA, et al. Estimating the global clinical burden of Plasmodium falciparum malaria in 2007. PLoS Med 7:e1000290.

13 Guerra CA, Howes RE, Patil AP, Gething PW, Van Boeckel TP, Temperley WH, et al. The international limits and population at risk of Plasmodium vivax transmission in 2009. PLoS Negl Trop Dis 2010;4:e774.

14 Nadjm B, Amos B, Mtove G, Ostermann J, Chonya S, Wangai H, et al. WHO guidelines for antimicrobial treatment in children admitted to hospital in an area of intense Plasmodium falciparum transmission: prospective study. BMJ 340:c1350.

15 Mtove G, Amos B, von Seidlein L, Hendriksen I, Mwambuli A, Kimera J, et al. Invasive salmonellosis among children admitted to a rural Tanzanian hospital and a comparison with previous studies. PLoS One 2010;5:e9244.

16 Joshi R, Colford JM Jr, Reingold AL, Kalantri S. Nonmalarial acute undifferentiated fever in a rural hospital in central India: diagnostic uncertainty and overtreatment with antimalarial agents. Am J Trop Med Hyg 2008;78:393-9.

17 Anstey NM, Russell B, Yeo TW, Price RN. The pathophysiology of vivax malaria. Trends Parasitol 2009;25:220-7.

18 Tjitra E, Anstey NM, Sugiarto P, Warikar N, Kenangalem E, Karyana M, et al. Multidrug-resistant Plasmodium vivax associated with severe and fatal malaria: a prospective study in Papua, Indonesia. PLoS Med 2008;5:e128.

19 Rowland M, Rab MA, Freeman T, Durrani N, Rehman N. Afghan refugees and the temporal and spatial distribution of malaria in Pakistan. Soc Sci Med 2002;55:2061-72.

20 Kolaczinski J, Graham K, Fahim A, Brooker S, Rowland M. Malaria control in Afghanistan: progress and challenges. Lancet 2005;365:1506-12

21 Brooker S, Leslie T, Kolaczinski K, Mohsen E, Mehboob N, Saleheen S, et al. Spatial epidemiology of Plasmodium vivax, Afghanistan. Emerg Infect Dis 2006;12:1600-2.

22 Peters DH, Noor AA, Singh LP, Kakar FK, Hansen PM, Burnham G. A balanced scorecard for health services in Afghanistan. Bull World Health Organ 2007;85:146-51.

23 Yeboah-Antwi K, Pilingana P, Macleod WB, Semrau K, Siazeele K, Kalesha P, et al. Community case management of fever due to malaria and pneumonia in children under five in Zambia: a cluster randomized controlled trial. PLoS Med 2010;7:e1000340.

24 Reyburn H, Mbatia R, Drakeley C, Carneiro I, Mwakasungula E, Mwerinde O, et al. Overdiagnosis of malaria in patients with severe febrile illness in Tanzania: a prospective study. BMJ 2004:329:1212

25 Ansah EK, Narh-Bana S, Epokor M, Akanpigbiam S, Quartey AA, Gyapong J, et al. Rapid testing for malaria in settings where microscopy is available and peripheral clinics where only presumptive treatment is available: a randomised controlled trial in Ghana. BMJ 2010;340:c930.

26 Bailey MS, Trinick TR, Dunbar JA, Hatch R, Osborne JC, Brooks TJ, et al. Undifferentiated febrile illnesses amongst British troops in Helmand, Afghanistan. J R Army Med Corps 2011:157:150-5.

27 Wallace MR, Hale BR, Utz GC, Olson PE, Earhart KC, Thornton SA, et al. Endemic infectious diseases of Afghanistan. Clin Infect Dis 2002;34(Suppl 5):S171-207.

28 Mtove G, Hendriksen IC, Amos B, Mrema H, Mandia V, Manjurano A, et al. Treatment guided by rapid diagnostic tests for malaria in Tanzanian children: safety and alternative bacterial diagnoses. Malar J 2011;10:290.

29 Berkley JA, Maitland K, Mwangi I, Ngetsa C, Mwarumba S, Lowe BS, et al. Use of clinical syndromes to target antibiotic prescribing in seriously ill children in malaria endemic area: observational study. BMJ 2005;330:995-9.

30 Brent AJ, Ahmed I, Ndiritu M, Lewa P, Ngetsa C, Lowe B, et al. Incidence of clinically significant bacteraemia in children who present to hospital in Kenya: community-based observational study. Lancet 2006:367:482-8.

31 Chandler $\mathrm{Cl}$, Jones C, Boniface G, Juma K, Reyburn H, Whitty CJ. Guidelines and mindlines: why do clinical staff over-diagnose malaria in Tanzania? A qualitative study. Malar J 2008;7:53.

32 Chandler $\mathrm{Cl}$, Whitty CJ, Ansah EK. How can malaria rapid diagnostic tests achieve their potential? A qualitative study of a trial at health facilities in Ghana. Malar J 9:95.

33 Chandler $\mathrm{Cl}$, Chonya S, Boniface G, Juma K, Reyburn H, Whitty CJ. The importance of context in malaria diagnosis and treatment decisions-a quantitative analysis of observed clinical encounters in Tanzania. Trop Med Int Health 2008;13:1131-42.

34 Wongsrichanalai C, Barcus MJ, Muth S, Sutamihardja A, Wernsdorfer WH. A review of malaria diagnostic tools: microscopy and rapid diagnostic test (RDT). Am J Trop Med Hyg 2007;77(6 Suppl):119-27.

35 Ashton RA, Kefyalew T, Tesfaye G, Counihan H, Yadeta D, Cundill B, et al. Performance of three multi-species rapid diagnostic tests for diagnosis of Plasmodium falciparum and Plasmodium vivax malaria in Oromia Regional State, Ethiopia. Malar J 9:297.

36 Acerra JR, Iskyan K, Qureshi ZA, Sharma RK. Rebuilding the health care system in Afghanistan: an overview of primary care and emergency services. Int J Emerg Med 2009;2:77-82

Accepted: 30 May 2012

\section{Cite this as: BMJ 2012;345:e4389}

This is an open-access article distributed under the terms of the Creative Commons Attribution Non-commercial License, which permits use, distribution, and reproduction in any medium, provided the original work is properly cited, the use is non commercial and is otherwise in compliance with the license. See: http://creativecommons.org/licenses/by$\mathrm{nc} / 2.0 /$ and http://creativecommons.org/licenses/by-nc/2.0/legalcode. 


\section{What is already known on this topic}

Global guidelines for malaria recommend treatment with malaria drugs only when a diagnostic test positively identifies malaria parasites in the patient's blood

In African settings, however, many patients are treated for malaria even when the diagnostic test result is negative

The extent of this problem is relatively unknown in south and central Asia where more than two billion people live in areas at risk of malaria

\section{What this study adds}

Overdiagnosis and mistreatment of patients negative for malaria is widespread in this part of Asia

Misdiagnosis and treatment is caused by inaccurate microscopy and the clinicians' tendency to treat with malaria drugs even when a test result is negative

Falciparum malaria is poorly targeted with effective drugs, and other potentially fatal causes of fever are missed even when a parasite based diagnosis is available

\section{Tables}

Table 1/ Description of clinics included in study, showing clinic type and diagnostic approach, by Afghan province

\begin{tabular}{lccc}
\hline Characteristics & North & East & Total \\
Transmission of parasites & & High & \\
\hline No of clinics: & 10 & 5 & 15 \\
\hline Basic Health Centres & 0 & 7 & 7 \\
\hline Comprehensive Health Centres & & \\
\hline Diagnostic method: & $10^{*}$ & 0 & 10 \\
\hline Clinical & $5^{*}$ & 5 \\
\hline New microscopy & 0 & 0 & 12 \\
\hline Established microscopy & 3043 & 5805 & 4549 \\
\hline Average No of attendees registered during study period, per clinic & & 5 \\
\hline
\end{tabular}

*Five clinics introduced microscopy in August 2009, part way through the study. 
Table 2| Characteristics of patients at enrolment according to type of diagnosis and Afghan province. Values are numbers (percentages) of patients unless stated otherwise

North

Characteristics

Clinical diagnosis $(n=418)$ New microscopy $(n=213)$ East: established microscopy $(n=1750)$

No enrolled:

\begin{tabular}{|c|c|c|c|}
\hline Comprehensive Health Centres & - & - & 958 \\
\hline Basic Health Centres & 418 & 213 & 792 \\
\hline$\%$ male & $147(35)$ & $89(42)$ & $784(44.8)$ \\
\hline Mean (SD) age (years) & $23.6(14)$ & $22.9(15)$ & $13.5(11.1)$ \\
\hline \multicolumn{4}{|l|}{ Age group: } \\
\hline $0-5$ & $27(6)$ & $17(8)$ & $355(20.3)$ \\
\hline $6-10$ & $32(8)$ & $24(11)$ & $614(35.1)$ \\
\hline $11-15$ & $71(17)$ & $36(17)$ & $250(14.3)$ \\
\hline $16-20$ & $102(24)$ & $45(21)$ & $247(14.1)$ \\
\hline $21-40$ & $138(33)$ & $69(32)$ & $241(13.8)$ \\
\hline$>40$ & $47(11)$ & $22(10)$ & $43(2.5)$ \\
\hline \multicolumn{4}{|l|}{ Clinician providing treatment: } \\
\hline Doctor & $376(90)$ & $147(69)$ & $1528(87.3)$ \\
\hline Nurse or midwife & $42(10)$ & $66(31)$ & $222(12.7)$ \\
\hline \multicolumn{4}{|c|}{ Average No (range) of patients enrolled at each clinic: } \\
\hline Comprehensive Health Centres & - & - & $137(70-213)$ \\
\hline Basic Health Centres & $42(25-76)$ & $43(11-65)$ & $95(29-209)$ \\
\hline
\end{tabular}


Table 3| Number and proportion of patients with suspected malaria who were accurately treated with malaria drugs measured against result of reference blood slide. Values are number affected/total number in group (percentage) unless stated otherwise

\begin{tabular}{|c|c|c|c|}
\hline \multirow[b]{2}{*}{ Variables } & \multicolumn{2}{|c|}{ North } & \multirow[b]{2}{*}{ East: established microscopy } \\
\hline & Clinical diagnosis & New microscopy & \\
\hline Reference slide positive for malaria & $1 / 415(0.2)$ & $0 / 202$ & $471 / 1740(27.1)$ \\
\hline \multicolumn{4}{|l|}{ Parasites detected in reference slide: } \\
\hline Plasmodium vivax & 1 & - & $465(98.7)$ \\
\hline Plasmodium falciparum & 0 & - & $6(1.2)$ \\
\hline Coinfection with $P$ vivax and $P$ falciparum & 0 & - & $1(0.2)^{*}$ \\
\hline \multicolumn{4}{|l|}{ Treated correctly: } \\
\hline According to reference slide result & $3 / 415(1)$ & $127 / 202(63)$ & $1056 / 1740(60.7)$ \\
\hline According to clinic slide result & - & 159/193 (82) & 1383/1663 (83.2) \\
\hline \multicolumn{4}{|l|}{ Treatment } \\
\hline \multicolumn{4}{|l|}{ Malaria status: } \\
\hline Negative, given malaria drugs & $412 / 414(99)$ & 75/202 (37) & $645 / 1269(50.8)$ \\
\hline Positive, not given correct malaria drugs & 0 & - & $29 / 471(6.2)$ \\
\hline \multicolumn{4}{|l|}{ Positive test result: } \\
\hline$P$ falciparum, treated with SP plus AS & - & - & $1 / 6(16.7)$ \\
\hline$P$ vivax, treated with $\mathrm{CQ} \dagger$ & $1(100)$ & - & $432 / 465(92.9)$ \\
\hline Mixed infection, treated with SP plus AS & - & - & 0 \\
\hline \multicolumn{4}{|l|}{ Negative test result: } \\
\hline$P$ falciparum, treated with SP plus AS & - & - & $3 / 1740(0.2)$ \\
\hline$P$ vivax, treated with $\mathrm{CQ} \dagger$ & $408 / 414(99)$ & $72 / 202(36)$ & $630 / 1274(49.4)$ \\
\hline Malaria, treated with SP plus CQ & $139 / 414(34)$ & $21 / 202(10)$ & $222 / 1269(17.5)$ \\
\hline \multicolumn{4}{|l|}{ Antibiotics prescribed: } \\
\hline Positive for malaria & 0 & - & $20 / 471(4.3)$ \\
\hline Negative for malaria & $47 / 414(11)$ & $103 / 202(60)$ & $342 / 1269(27.0)$ \\
\hline
\end{tabular}

$\mathrm{SP}=$ sulfadoxine-pyrimethamine; $\mathrm{AS}=$ artesunate; $\mathrm{CQ}=$ chloroquine.

*Patient also included and evaluated as being positive for $P$ vivax and $P$ falciparum, separately.

†Including patients treated with SP plus CQ. 
Table 4| Univariate and multivariate analysis of factors associated with accurate treatment with malaria drugs in patients with suspected malaria. Values are number accurately treated/number in group (percentage) unless stated otherwise

\begin{tabular}{|c|c|c|c|c|c|}
\hline Variables & Accurately treated & Odds ratio $(95 \% \mathrm{Cl})$ & $P$ value & Adjusted odds ratio $(95 \% \mathrm{Cl})$ & $P$ value \\
\hline \multicolumn{6}{|l|}{ Diagnostic method*: } \\
\hline Clinical & $3 / 412(0.7)$ & 1 & & 1 & \\
\hline New microscopy & 127/202 (62.9) & 232 (72 to 750$)$ & $<0.001$ & 229 (71 to 743$)$ & $<0.001$ \\
\hline Established microscopy & $1056 / 1739(60.7)$ & 212 (68 to 664$)$ & $<0.001$ & 102 (32.3 to 323$)$ & $<0.001$ \\
\hline \multicolumn{6}{|l|}{ Diagnosis*: } \\
\hline Malaria & $433 / 471$ (91.9) & 1 & & 1 & \\
\hline Not malaria & $753 / 1885(40.0)$ & 0.06 (0.04 to 0.08$)$ & $<0.001$ & 0.07 (0.05 to 0.1$)$ & $<0.001$ \\
\hline \multicolumn{6}{|l|}{ Patients' sext: } \\
\hline Male & $546 / 1099(54.1)$ & 1 & & 1 & \\
\hline Female & 640/1347 (47.5) & $0.8(0.7$ to 0.9$)$ & 0.002 & $1.0(0.8$ to 1.2$)$ & 1.0 \\
\hline \multicolumn{6}{|l|}{ Age group*: } \\
\hline $0-5$ & 250/396 (63.1) & 1 & & 1 & \\
\hline $6-10$ & 358/666 (53.8) & $0.7(0.5$ to 0.9$)$ & 0.003 & $0.6(0.4$ to 0.8$)$ & $<0.001$ \\
\hline $11-15$ & $176 / 352(50.0)$ & $0.6(0.4$ to 0.8$)$ & $<0.001$ & 0.9 (0.6 to 1.3$)$ & 0.6 \\
\hline $16-20$ & $177 / 390(45.4)$ & 0.5 (0.4 to 0.6$)$ & $<0.001$ & $1.1(0.8$ to 1.6$)$ & 0.6 \\
\hline $21-40$ & $188 / 440(42.7)$ & $0.4(0.3$ to 0.6$)$ & $<0.001$ & $1.1(0.8$ to 1.6$)$ & 0.6 \\
\hline$>40$ & $37 / 112(33.0)$ & $0.3(0.2$ to 0.4$)$ & $<0.001$ & $0.8(0.4$ to 1.4$)$ & 0.3 \\
\hline \multicolumn{6}{|l|}{ Clinician providing treatment ${ }^{*}$ : } \\
\hline Doctor & 979/2034 (48.1) & 1 & & 1 & \\
\hline Nurse or midwife & 207/322 (64.3) & $1.9(1.5$ to 2.5$)$ & $<0.001$ & $2.1(1.6$ to 2.9$)$ & $<0.001$ \\
\hline \multicolumn{6}{|l|}{ Clinic type*: } \\
\hline Basic Health Centre & $531 / 1405(37.8)$ & 1 & & 1 & \\
\hline Comprehensive Health Centre & 655/951 (68.9) & 3.6 (3.1 to 4.3$)$ & $<0.001$ & $2.4(1.9$ to 2.9$)$ & $<0.001$ \\
\hline
\end{tabular}


Table 5| Accuracy of clinic level diagnosis compared with double or triple read reference slides. Values are number/number in group (percentage) unless stated otherwise

\begin{tabular}{lccc} 
Variables & North: new microscopy & East: established microscopy & Clinician in north* \\
False positive result & $39 / 193(20.2) \dagger$ & $329 / 1212(27.1)$ & $412 / 413(99.8)$ \\
\hline False negative result & - & $47 / 451(10.4)$ & $0 / 1$ \\
\hline Sensitivity, \% (95\% Cl) & - & $89.6(88.1$ to 91.1$)$ & 100 \\
\hline Specificity, \% (95\% Cl) & $79.8(73.4$ to 85.2) & $72.9(70.7$ to 75.0$)$ & $0.2(0$ to 0.7$)$ \\
\hline Positive predictive value & - & $55.1(52.7$ to 57.5$)$ & $0.2(0$ to 0.7$)$ \\
\hline Negative predictive value & - & $95.0(93.9$ to 96.0$)$ & 100
\end{tabular}

*Against clinicians diagnosis based on signs and symptoms alone.

$\dagger$ New compared with established microscopy: Fisher's exact, $\mathrm{P}=0.02$. 
Table 6| Accuracy of clinicians' prescription of malaria drugs against clinic slide results, stratified by status of prescriber. Values are number/number in group (percentage) unless stated otherwise

Doctors

Nurses or midwives

Variables

North: new microscopy East: established microscopy North: new microscopy East: established microscopy

Negative result, treated (false positive) $25 / 109(22.9)^{*}$ $254 / 795(31.9)^{*}$ $7 / 45(15.6)$ $16 / 140(11.4)$

Positive result, not treated (false negative) $2 / 27(7.4)$ $1 / 658(0.2)$ $0 / 12$ $0 / 75$

Sensitivity, \% $(95 \% \mathrm{Cl})$

$92.6(88.2$ to 97.0$)$

99.9 (99.7 to 100$)$

100

Specificity, \% $(95 \% \mathrm{Cl}) \quad 77.1(70.0$ to 84.1$)$

$66.7(64.3$ to 69.1$)$

84.4 (75.0 to 93.9$)$

$88.6(84.3$ to 92.8$)$

*Doctors compared with nurses: in north Fisher's exact, $\mathrm{P}=0.3$; in east Fisher's exact, $\mathrm{P}<0.001$. 


\section{Figure}

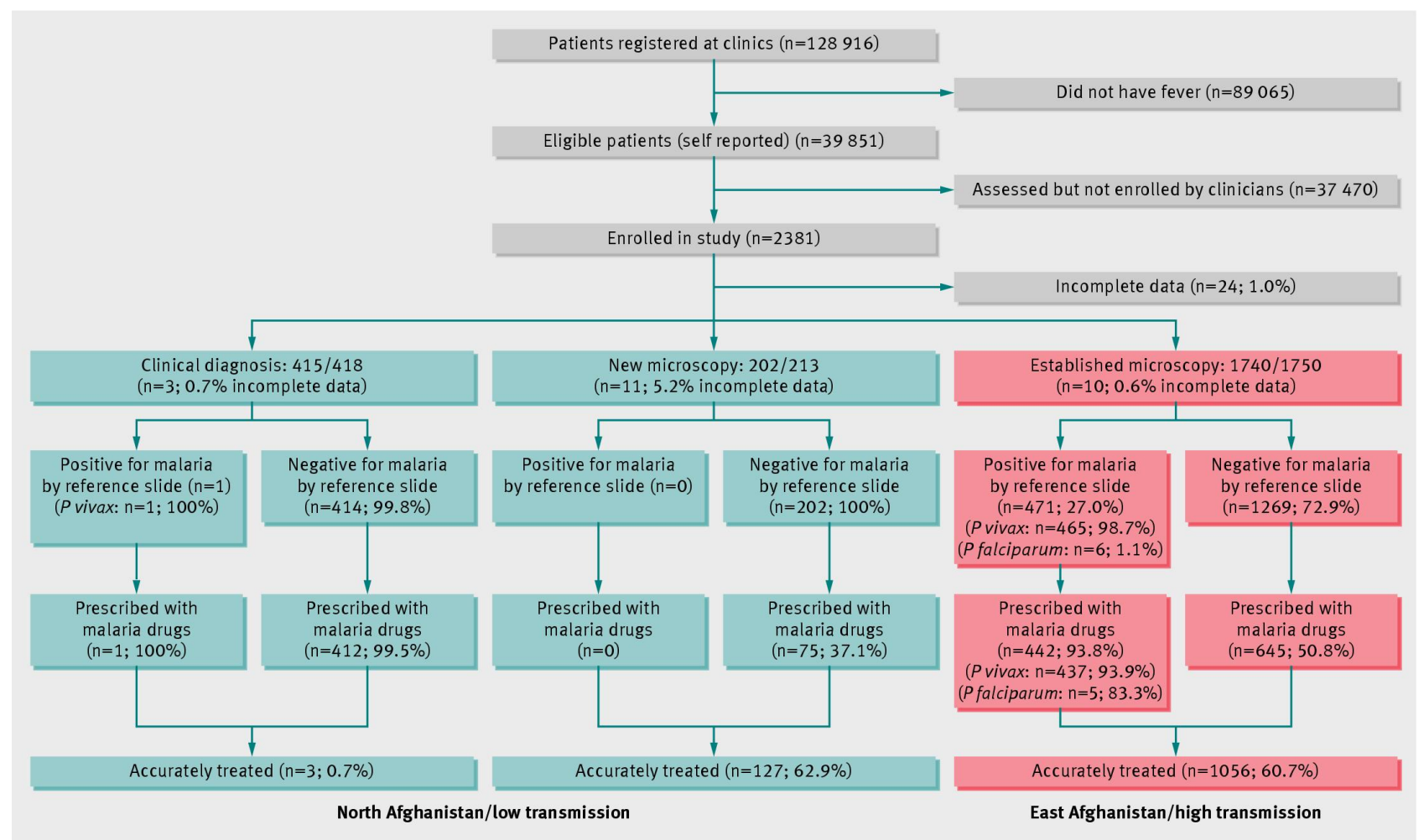

Patient flow through study. Accurately treated is defined as patients with malaria parasites given appropriate malaria drugs and those without malaria parasites not given malaria drugs 